\title{
Chest wall-parallel vs. conventional subclavian venous catheterization in cancer chemotherapy: A comparison of complication rates
}

\author{
LIBO LI ${ }^{1}, \mathrm{HANG} \mathrm{LI}^{1}$, LINLI XU ${ }^{2}$ and $\mathrm{LEI} \mathrm{SONG}^{3}$ \\ ${ }^{1}$ Department of Oncology, Guizhou Provincial People's Hospital, Guiyang, Guizhou 550002; \\ ${ }^{2}$ Department of Oncology, Beijing Renhe Hospital, Beijing 102600; ${ }^{3}$ Department of Clinical Laboratory \\ Guizhou Medical University Affiliated Hospital, Guiyang, Guizhou 550002, P.R. China
}

Received May 12, 2017; Accepted August 21, 2017

DOI: $10.3892 / \mathrm{ol} .2017 .6923$

\begin{abstract}
The incidence of complications such as pneumothorax and hematoma between the chest wall-parallel and conventional subclavian venous catheterization in cancer chemotherapy was compared. From December 2011 to March 2016, a total of 314 patients undergoing chemotherapy for cancer in the Guizhou Provincial People's Hospital were assigned to either the Chest Wall-parallel $(n=155)$ or the conventional subclavian venous catheterization group $(n=159)$ in order to observe the primary success rate for catheterization and to assess the incidence of complications such as pneumothorax, hemothorax, hematoma, and internal jugular venous injury. The primary success rates for catheterization were not significantly different between the conventional and chest wall-parallel subclavian venous catheterization groups $(94.3 \%$ vs. $96.8 \%, \mathrm{P}>0.05)$, with a total catheterization success rate of $100 \%$ in both groups. However, the incidence of pneumothorax was significantly different between the groups $(6.29 \%$ in conventional vs. $0 \%$ in chest wall-parallel subclavian venous catheterization group, $\mathrm{P}<0.05)$. Therefore, compared to conventional subclavian venous catheterization, the chest wall-parallel approach could reduce the risk of or even totally prevent pneumothorax and other venipunctures and is, thus, a relatively safe and effective technique that could have wide applications in clinical settings.
\end{abstract}

\section{Introduction}

Cancer chemotherapy is a long-term process in which most chemotherapeutics are intravenously administered. However,

Correspondence to: $\mathrm{Dr} \mathrm{Hang} \mathrm{Li}$, Department of Oncology, Guizhou Provincial People's Hospital, 83 Zhongshan East Road, Guiyang, Guizhou 550002, P.R. China

E-mail: lihang4412@163.com

Key words: chemotherapy, chest wall-paralleled, pneumothorax, subclavian venous catheterization the majority of chemotherapeutics induce thrombophlebitis, which causes liquid medications to easily extravasate through the small-caliber peripheral vessels to cause local tissue swelling, erythema, and even necrosis which requires surgical treatment. In contrast, the central vein has a relatively large lumen and high blood flow velocity, which decreases the stimulation of drugs to blood vessels and the incidence of thrombophlebitis and extravasation (1). The central venous route also helps avoid pain that is associated with repeated venipunctures in chemotherapy patients, which remarkably improve treatment safety and the quality of life in cancer patients receiving long-term intravenous infusion. It also allows for the design of more complex and effective multiple drug regimens by clinicians. Early in 2006, the Infusion Nurses Society stipulated the use of the central venous route device in chemotherapy (2). With the popularization of longterm indwelling venous catheters, the most commonly used devices include subclavian/internal jugular/femoral venous catheters and peripherally inserted central catheters (PICCs), among which the subclavian venous catheter is the easiest to fix (3), the least liable to dislodge, and has the lowest failure rate (4). Thus, it is widely applied in clinical settings. However, conventional venipuncture is prone to complications such as pneumothorax, hematoma, and internal jugular venous injury. One study has been reported that pneumothorax and catheter malposition occur in 3.3 and $9.1 \%$ patients, respectively (5). This study aims to compare the incidence of complications such as pneumothorax, hematoma, and catheter malposition following chest wall-parallel and conventional subclavian venous catheterization, for applications in clinical practice.

\section{Patients and methods}

Patient information. Three hundred and fourteen patients (196 males, 118 females; age, 25-78 years) who were undergoing central venous catheterization for chemotherapy in the Department of Oncology, Guizhou Provincial People's Hospital between December 2011 and March 2016 were retrospectively analyzed for this study. Conventional and chest wall-parallel subclavian venous catheterization was performed in 159 and 155 patients, respectively. All patients 


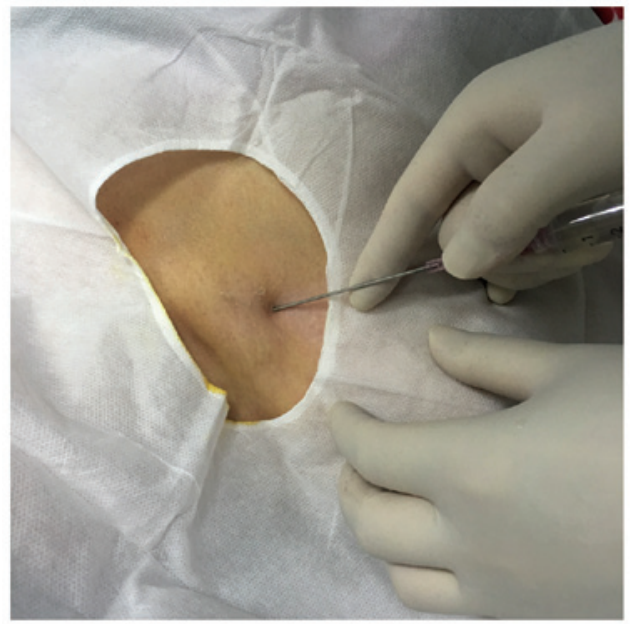

Figure 1. Conventional subclavian venous catheterization procedure (sagittal view).

had normal coagulation function and peripheral platelet count before catheterization. The study protocol was approved by the ethics committees of the Guizhou Provincial People's Hospital, and written informed consent was obtained from all participants.

Routine catheterization procedure. The patient was placed in the supine position, with the head turned to the left side. The neck, chest, and shoulders were routinely disinfected and draped in a sterile manner. Under local anesthesia, a puncture needle held under negative pressure and pointing to the suprasternal notch at an angle of $30-45^{\circ}$ against the chest wall was inserted $1 \mathrm{~cm}$ below the site, $1-2 \mathrm{~cm}$ proximal to the right clavicle midpoint, or at the distal one-third point between the midpoint and the proximal end of the right clavicle. During the insertion, the operator drew blood until dark red blood was extracted, which indicated the successful entry of the needle into the subclavian vein. Then, the patient was asked to hold or slow his/her breathing and the syringe was removed, followed by a quick introduction of a guide wire through the puncture needle into the subclavian vein. Subsequently, the guide wire was pushed for another $10-15 \mathrm{~cm}$ to reach the superior vena cava. Next, the puncture needle was removed and a catheter was introduced over the guide wire into the superior vena cava. The guide wire was removed, and the return of venous blood confirmed the entry of the catheter into the vein. Normal heparinized saline solution was injected in all the lumens of the catheter, which was then secured with silk sutures to the skin. Post-operative chest radiography was performed in order to detect the catheter position and the occurrence of complications such as pneumothorax. The catheterization procedure is illustrated in Fig. 1.

Chest wall-parallel subclavian venous catheterization procedure. Routine disinfection and draping techniques were performed. A puncture needle that was attached to a syringe filled with normal heparinized saline solution was inserted $0.5-1 \mathrm{~cm}$ below the distal one-third point of the clavicle, with the needle tip pointing to the right supraclavicular fossa. When the needle was inserted into the skin, puncture needle was

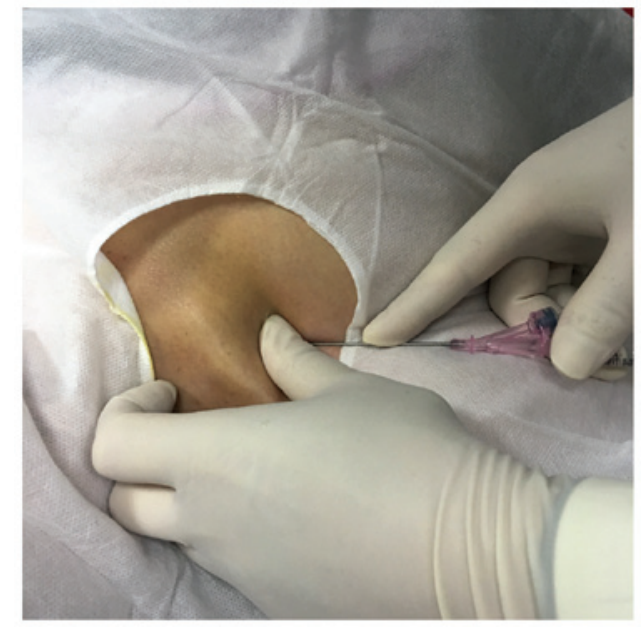

Figure 2. Chest wall-parallel subclavian venous catheterization procedure (sagittal view)

immediately put parallel to the chest wall, and then pressed so that it subsides $1-2 \mathrm{~cm}$, which allows it to be close to the chest wall and pushes it to the right supraclavicular fossa direction advance. The operator attempted to withdraw blood during the insertion, and the smooth extraction of blood indicated entry into the subclavian vein. The patient was then requested to hold or slow his/her breathing and the syringe was removed, which was followed by the quick insertion of a guide wire through the puncture needle into the subclavian vein. Subsequently, the guide wire was pushed for another $10-15 \mathrm{~cm}$ in order to reach the superior vena cava. Next, the puncture needle was removed and a catheter was inserted over the guide wire up to the superior vena cava. The guide wire was removed, and the return of venous blood confirmed the entry of the catheter into the vein. Normal heparinized saline solution was injected into the catheter lumen and the catheter was secured with silk sutures to the skin. Post-operative chest radiography was performed in order to observe the catheter position and to check for complications such as the pneumothorax. The procedure is illustrated in Fig. 2.

Evaluation methods. The principal evaluation parameters included the primary success rate for catheterization and the incidence of complications such as pneumothorax, hemothorax, hematoma, and internal jugular venous injury, as shown on post-operative radiograph.

Statistical analysis. The complication rates were calculated and data were analyzed using SPSS software (version 19.0). The Chi-squared test was used for categorical variables. $\mathrm{P}<0.05$ was considered to indicate a statistically significant difference.

\section{Results}

The 314 tumor patients included 136 patients with colon cancer, 135 with rectal cancer, and 43 with cervical cancer. Among the patients, 159 patients underwent conventional subclavian venous catheterization, which demonstrated primary and secondary success rates of $94.3 \%(150 / 159)$ and $5.7 \%(9 / 159)$, 


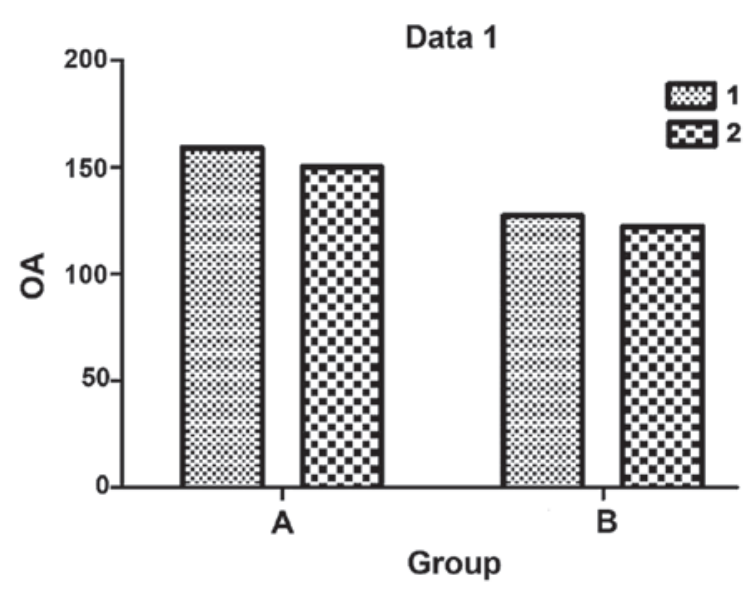

Figure 3. Comparison of catheterization outcomes between the groups.

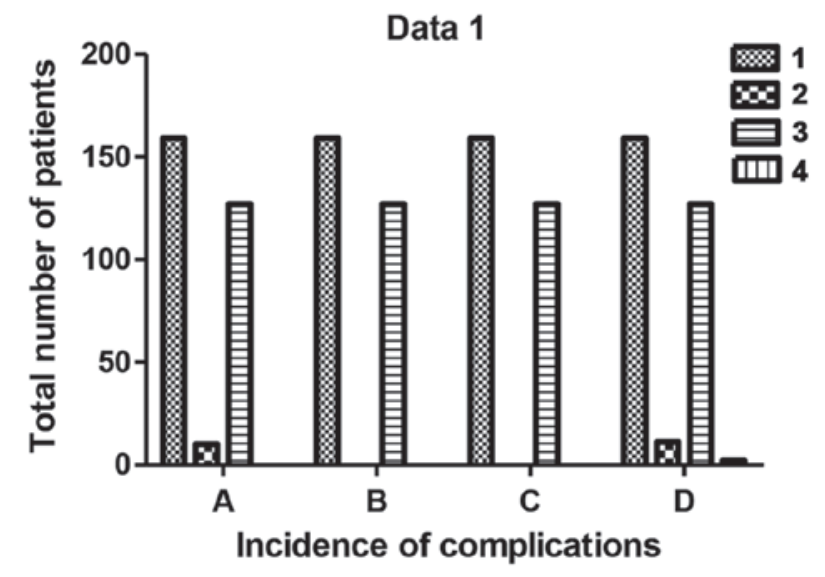

Figure 4. Comparison of complications between the groups.
Table I. Comparison of primary success rates for catheterization between the groups.

\begin{tabular}{lcc}
\hline Group & $\begin{array}{c}\text { Conventional } \\
\text { subclavian } \\
\text { venous } \\
\text { catheterization }\end{array}$ & $\begin{array}{c}\text { Chest wall-paralleled } \\
\text { subclavian } \\
\text { venous } \\
\text { catheterization }\end{array}$ \\
\hline Total success rate & $100 \%(159)$ & $100 \%(127)$ \\
Primary success rate & $94.3 \%(150)$ & $96.1 \%(122)$ \\
\hline
\end{tabular}

Both P-values $>0.05$. 1, Conventional subclavian venous catheterization group; 2, Chest wall-parallel subclavian venous catheterization group.

respectively. On the other hand, 155 patients underwent chest wall-parallel subclavian venous catheterization, which demonstrated primary and secondary success rates of $96.8 \%$ (150/155) and 3.2\% (5/155), respectively (Table I and Fig. 3).

Pneumothorax occurred in $10(6.29 \%)$ patients in the conventional subclavian venous catheterization group, while it was not observed in any patients in the chest wall-parallel subclavian venous catheterization group $(\mathrm{P}<0.005)$. The incidence of catheter malposition was significantly different between the groups [11 patients $(6.92 \%)$ vs. 2 patients $(1.29 \%)$; $\mathrm{P}<0.005]$. Neither of the catheterization techniques induced severe complications such as hemothorax or hematoma (Table II and Fig. 4).

\section{Discussion}

The subclavian vein, which is a direct branch of the axillary vein, is surrounded by the clavicle at the anterior aspect, by the anterior scalene muscle, phrenic nerve, subclavian artery, brachial plexus, pleural cupula, and (on the left side) the thoracic duct at the posterior aspect, and by the clavicle, first rib, and anterior scalene muscle at the anterior superior aspect (6). The subclavian vein measures approximately $2 \mathrm{~cm}$ in diameter in adults and is characterized by a large lumen, a lack of venous valves, less pressure, ease of fixation, and a low possibility of dislodgement, and is thus safe and reliable for
Table II. Comparison of complication rates between the groups.

\begin{tabular}{lccc}
\hline Group & $\begin{array}{c}\text { Conventional } \\
\text { subclavian } \\
\text { venous } \\
\text { catheterization }\end{array}$ & $\begin{array}{c}\text { Chest } \\
\text { wall-parallel } \\
\text { subclavian } \\
\text { venous } \\
\text { catheterization }\end{array}$ & P-value \\
\hline $\begin{array}{l}\text { Pneumothorax } \\
\text { Hemothorax }\end{array}$ & $6.29 \%(10)$ & 0 & 0.004 \\
Hematoma & 0 & 0 & 0 \\
Internal jugular & $6.92 \%(11)$ & 0 & 0 \\
venous injury & & $1.57 \%(2)$ & 0.031 \\
\hline
\end{tabular}

catheterization (7). The subclavian vein is a common vessel for interventional techniques.

The pleural cupula, which is located $2-3 \mathrm{~cm}$ higher than the proximal one-third point of the clavicle, is anteriorly connected to the subclavian artery and its branches, phrenic nerve, and subclavian vein. The subclavian vein and internal jugular vein meet in the inside of the front edge of thoracic outlet, behind the breastbone handle form around head arm vein, and form in the sternum rear left and right brachiocephalic vein. Thus, a subclavian venipuncture can easily go into internal jugular vein. Subclavian vein cannulation (SCV) via the traditional approach has become used less in comparison to the ultrasound (US) guided subclavian vein catheterization.

Conventionally, a puncture needle held under negative pressure is inserted $1-2 \mathrm{~cm}$ proximal to the midpoint of the right clavicle or $1 \mathrm{~cm}$ below the distal one-third point between the midpoint of the clavicle and the sternal edge, with the tip pointing interiorly and upwards to the suprasternal notch at an angle of $30-45^{\circ}$ against the chest wall, until the return of venous blood is observed. However, the puncture techniques fail to take the thoracic shape and the tissues adjacent to the subclavian vein into consideration. The insertion angle of $30-45^{\circ}$ may lead to pneumothorax if the needle passes through the subclavian vein into the pleural cupula. According to the current Swedish clinical guidelines on central venous catheterization, subclavian 
venous catheterization has a very high risk for pneumothorax and hematoma (8).

Currently, ultrasound guidance is the only standard of care for internal jugular vein catheterization in many centers, and its use is strongly recommended by clinical practice guidelines. A meta-analysis conducted for a longer period of time looking at both ultrasound-guided cannulation versus the landmark technique to determine overall failed catheterizations and catheter malposition revealed no significant difference between the ultrasound and landmark technique. The use of ultrasound was associated with a significant decrease in overall complications, and variations existed in both operator experience and ultrasound approach (9). Ultrasound guidance is the only standard of care for internal jugular vein catheterization in many centers, and its use is strongly recommended by clinical practice guidelines, however, there were no differences in failed catheterization rates between landmark and ultrasound use. There are variations present in operator experience and the ultrasound approach. Five of these reports used a dynamic 2D ultrasound and two reports used Doppler ultrasound. The meta-analysis of these seven studies demonstrate that there is no reduction in catheter malposition with ultrasound guidance (10). US guidance reduces the number of mechanical complications, the number of catheter placement failures and the time required for insertion (11).

Therefore, several modified methods for subclavian venipuncture have been proposed in China, however, they frequently involve adjustments of the puncture site and direction. Nevertheless, an accurate adjustment of the insertion angle has not yet been reported. As in the conventional puncture method, these modifications are limited to the operator's expertise. If the catheter is inserted too deep, then it will penetrate into the pleural cupula, leading to pneumothorax. Based on the anatomical structures of the human thorax and the root of the neck, chest wall-parallel subclavian venipuncture considers the fact that the subclavian artery, phrenic nerve, and pleural cupula are located behind the subclavian vein. Hence, inserting the catheter parallel to the chest wall only injures the muscles and reduces or avoids damage to the pleural cupula, thus preventing pneumothorax. It is important to recognize, however, that adverse events may also occur under RTUS-guidance.

Our study reveals that the primary success rates for catheterization were not significantly different between the two groups $(\mathrm{P}>0.05)$. There were no serious complications such as hemothorax, hematoma, and other venipunctures that occurred in either group. The incidence of pneumothorax and catheter malposition significantly differed between the chest wall-parallel and conventional subclavian venous catheterization groups $(\mathrm{P}<0.004$ and $\mathrm{P}=0.031$, respectively). Therefore, we conclude that chest wall-parallel subclavian venous catheterization does not significantly differ from conventional catheterization with regards to the success rate, however, the former is safer and more reliable. It can reduce, even avoid pneumothorax and reduce catheter malposition.

Our study reveals that the incidence of pneumothorax is $0 \%$, and the incidence of catheter malposition is $1.57 \%$. A meta-analysis reveals that the incidence of pneumothorax is $0.6 \%$ in all of the studies with ultrasound guidance, and the incidence of catheter malposition is $4.55 \%$. It also reveals that the catheter malposition occurrence rate is lower than that of ultrasound guidance, and there were no incidence of pneumothorax. However, there are only a small number of cases in this study, and therefore, future studies should include greater numbers. One study demonstrates that to use of real-time US guidance for infraclavicular placement of SCV catheters can lead to decreased mechanical complications and improved cannulation success (11). The alternative cannulations require advanced training and experience (12).

Our results suggest that the chest wall-parallel subclavian venous catheterization can help patients feel more secure during the therapeutic process; and it thus holds promise for widespread use in clinical settings.

\section{References}

1. Khor T, Anderson J and McRae P: Central venous thrombophlebitis diagnosed by computerized tomography scanning. Aust N Z J Surg 62: 820-822, 1992.

2. Infusion Nurses Society: Infusion nursing standards of practices. J Infus Nurs 29 (Suppl 1): S1-S92, 2006

3. Bourgeois FC, Lamagna P and Chiang VW: Peripherally inserted central catheters. Pediatr Emerg Care 27: 556-561, 562-563, 2011.

4. Biffi R, Orsi F, Pozzi S, Pace U, Bonomo G, Monfardini L, Della Vigna P, Rotmensz N, Radice D, Zampino MG, et al: Best choice of central venous insertion site for the prevention of catheter-related complications in adult patients who need cancer therapy: A randomized trial. Ann Oncol 20: 935-940, 2009.

5. Dauser B, Stopfer J, Ghaffari S and Herbst F: Subclavian vein puncture vs. surgical cut-down to the cephalic vein for insertion of totally implantable venous access ports. Eur Surg 44: 331-335, 2012.

6. Turcotte S, Dubé S and Beauchamp G: Peripherally inserted central venous catheters are not superior to central venous catheters in the acute care of surgical patients on the ward. World J Surg 30: 1605-1619, 2006.

7. Araujo CC, Lima MC and Falbo GH: Percutaneous subclavian central venous catheterization in children and adolescents: success, complications and related factors. J Pediatr (Rio J) 83: 64-70, 2007.

8. Frykholm P,Pikwer A, Hammarskjöld F, Larsson AT, Lindgren S, Lindwall R, Taxbro K, Oberg F, Acosta S and Akeson J; Swedish Society of Anaesthesiology and Intensive Care Medicine: Clinical guidelines on central venous catheterisation. Acta Anaesthesiol Scand 58: 508-524, 2014.

9. Lalu MM, Fayad A, Ahmed O, Bryson GL, Fergusson DA Barron CC, Sullivan P and Thompson C; Canadian Perioperative Anesthesia Clinical Trials Group: Ultrasound-guided subclavian vein catheterization: A systematic review and meta-analysis. Crit Care Med 43: 1498-1507, 2015.

10. Aribaş BK, Arda K, Aribaş O, Ciledağ N, Yoloğlu Z, Aktaş E, Seber T, Kavak S, Coşar Y, Kaygusuz H, et al: Comparison of subcutaneous central venous port via jugular and subclavian access in 347 patients at a single center. Exp Ther Med 4: 675-680, 2012.

11. Dietrich CF, Horn R, Morf S, Chiorean L, Dong Y, Cui XW, Atkinson NS and Jenssen C: Ultrasound-guided central vascular interventions, comments on the European Federation of Societies for Ultrasound in Medicine and Biology guidelines on interventional ultrasound. J Thorac Dis 8: E851-E868, 2016.

12. Rezayat T, Stowell JR, Kendall JL, Turner E, Fox JC and Barjaktarevic I: Ultrasound-Guided Cannulation: Time to Bring Subclavian Central Lines Back. West J Emerg Med 17: 216-221, 2016. 